\title{
Síndrome inflamatorio multisistémico en niños asociado al SARS-CoV-2
}

\author{
Multisystemic inflammatory syndrome in children \\ associated with SARS-CoV-2
}

\author{
Olga Gutierrez ${ }^{1}$, Sandra Prantte Romero ${ }^{1}$
}

\section{RESUMEN}

En general la afectación del Covid 19 en niños es leve o asintomática. Un porcentaje pequeño de pacientes requirieron ingreso a la Unidad de cuidados intensivos pediátricos. Se reporta el caso de un paciente pediátrico hospitalizado en Unidad de Cuidados Intensivos de un hospital pediátrico, en el contexto de la pandemia SARS$\mathrm{CoV}-2$, que requirió ingreso a la UCI por presentar un shock tóxico, con marcadores inflamatorios, reactantes de fase aguda elevados y manifestaciones clínicas que corresponden al Síndrome Inflamatorio Multisistémico descripto recientemente y relacionado a la infección por COVID-19.

Palabras claves: Covid 19, síndrome inflamatorio multisistémico, pediatría, shock tóxico.

\section{INTRODUCCIÓN}

El Coronavirus es un grupo de virus que coinfectan humanos y animales. Afecta el aparato respiratorio, gastrointestinal, hígado y SNC de los humanos, ganado, pájaros, ratones, murciélagos entre otros. En diciembre del 2019 se aísla una nueva variedad de coronavirus, en humanos, la OMS lo denomino Covid 19 y al virus SARS -CoV2, para diferenciarlo del SARS CoV 1 del $2002^{(1,2)}$.

\begin{abstract}
In general, Covid 19 infection in children is mild or asymptomatic. A small percentage of patients require admission to the Pediatric Intensive Care Unit. We report the case of a pediatric patient hospitalized in the Intensive Care Unit of a pediatric hospital, in the context of the SARS-CoV-2 pandemic, who required admission to the ICU due to toxic shock, with elevation of inflammatory markers and acute phase reactants, along with clinical manifestations that corresponded to the recently described Multisystemic Inflammatory Syndrome related to COVID-19infection.
\end{abstract}

Key words: Covid 19, multisystemic inflammatory syndrome, pediatrics, toxic shock.
En diciembre del 2019 se constata gran transmisibilidad interhumano del SARS CoV2. A partir de enero del 2020 se comprueba la transmisión en conglomerados familiares, entre ellos, la transmisión a los niños ${ }^{(3)}$.

La transmisión es más frecuentemente por vía respiratoria (gotas aerolizadas) y por contacto, reporte de casos y serie de casos no demostraron

\footnotetext{
${ }^{1}$ Hospital General Pediátrico Niños de Acosta Ñu. San Lorenzo, Paraguay. Correspondencia: Olga Gutierrez Correo: draolgagutierrez@gmail.com Conflicto de Interés: Los autores declaran no poseer conflicto de interés Recibido: 20/06/2020 Aceptado: 09/07/2020

Doi: https://doi.org/10.31698/ped.47022020012
} 
transmisión vertical. En China la extensión de la epidemia se dio al asistir los niños a la escuela. Fueron los niños generalmente asintomáticos, los impulsores de la diseminación de la infección en la familia y en $1 a^{(4,5)}$.

Las manifestaciones clínicas de la Covid 19 en niños son leves en la mayoría de los casos. Frecuentemente son asintomáticos y un pequeño porcentaje de casos desarrollan formas graves con ingreso a la unidad de cuidados intensivos pediátricos. Los casos sintomáticos presentan fiebre, tos seca y dificultad respiratoria (similar a los adultos). Con menor frecuencia rinorrea y congestión nasal. Puede presentarse otros síntomas como nauseas vómitos, dolor abdominal, diarrea. En general se recuperan en 1 a 2 semanas. Se reportan algunos casos graves que necesitaron ingresar a la UCIP en china ${ }^{(6)}$.

En la pandemia por SARS CoV 2, tanto en Europa como en EEUU se ha observado un incremento de casos de niños con manifestaciones multisistémicas inflamatorias que desarrollaron un cuadro similar a la Enfermedad de Kawasaki ${ }^{(7)}$.

Se presenta un caso de afectación inflamatoria multisistémica con características de la enfermedad de Kawasaki en un hospital pediátrico con PCR e IgG positivo para SARS CoV 2.

\section{CASO CLÍNICO}

Paciente escolar, sexo masculino, de 9 años, procedente de zona urbana, sin patologías de base ni internaciones previas, con obesidad como comorbilidad. Es remitido desde otro hospital para ingreso a la unidad de cuidados intensivos pediátricos con un cuadro de shock de posible origen infeccioso. Se encontraba en el 4to día del post operatorio, por abdomen agudo quirúrgico con fiebre, de posible origen apendicular.

Ingresa lucido, hemo dinámicamente inestable con marcada taquicardia, con mala perfusión periférica, con tendencia a la hipotensión, recibe expansión con cristaloides e inicia inotrópicos (Dobutamina más Noradrenalina) a bajas titulaciones con buena respuesta y se suspenden a las $48 \mathrm{hs}$.Presentaba taquipnea con requerimiento de oxígeno suplementario por mascarilla de alto flujo por 4 días. Ante acidosis metabólica recibió corrección de bicarbonato en 1 oportunidad. Buen ritmo diurético, perfil renal en rango. Se rotó a múltiple antibioticoterapia para cobertura de sepsis a foco pulmonar o enteral.

Presentaba además conjuntivitis no purulenta bilateral (Fig. 1 y 2), además de lesiones en piel tipo maculas eritematosas, en diana compatibles con eritema multiforme, que confluyen sobre todo en ambos flancos (Fig 3), y en menor medida en piernas, brazos y tórax, no pruriginosas (Fig.4 ,5 y 6), que se iniciaron en el post quirúrgico inmediato. La piel estaba seca y áspera en el rostro con pequeñas descamaciones. Mucosa oral, labios, palmas de manos y pies, sin particularidades; no se palpan ganglios superficiales.

Treinta días antes del ingreso presento cuadro febril con afectación respiratoria, con retorno de PCR positivo para SARS CoV 2. Tenía contacto estrecho con familiares con Covid 19. El cuadro fue leve y no requirió hospitalización.

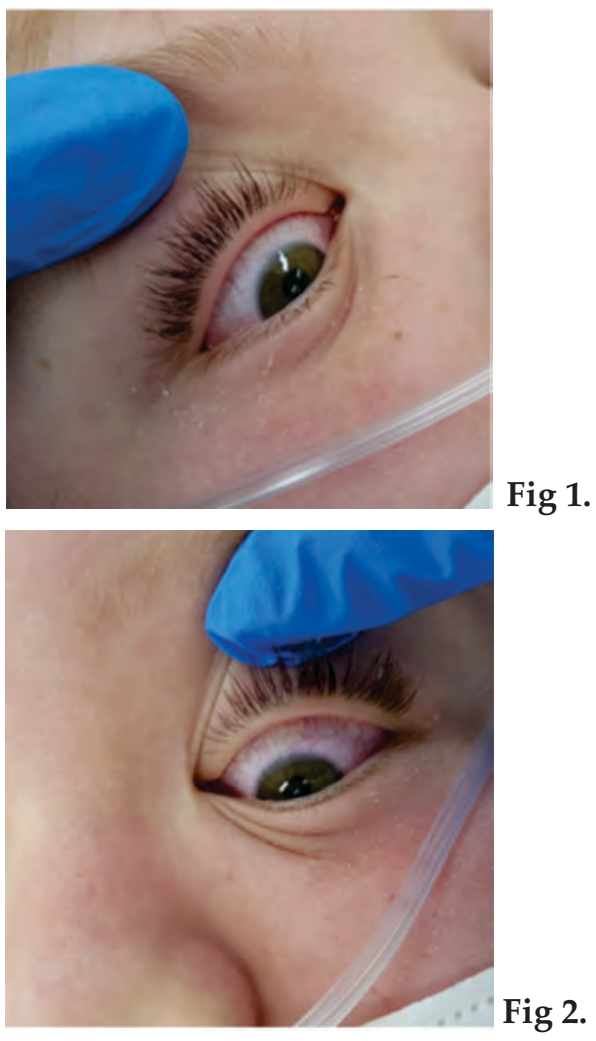

Fig 1 y 2. congestión de las conjuntivas, sin secreción. 


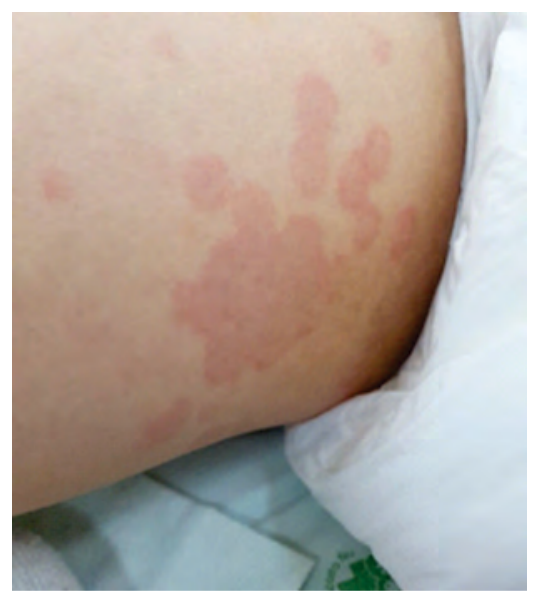

Fig 3. Placas numulares eritematosas, bien delimitadas, con centro violáceo, que coalescen formando placas mayores, en ambos flancos.

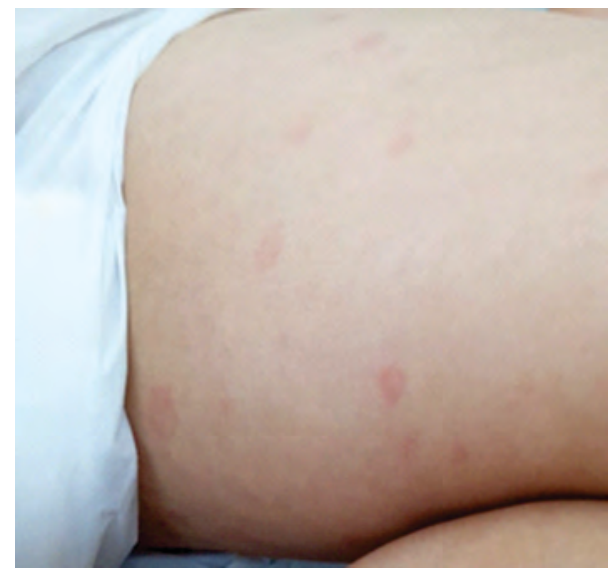

Fig 4. Placas numulares en ambos muslos, eritematosas, bien delimitadas, más pequeñas, patrón polimórfico.
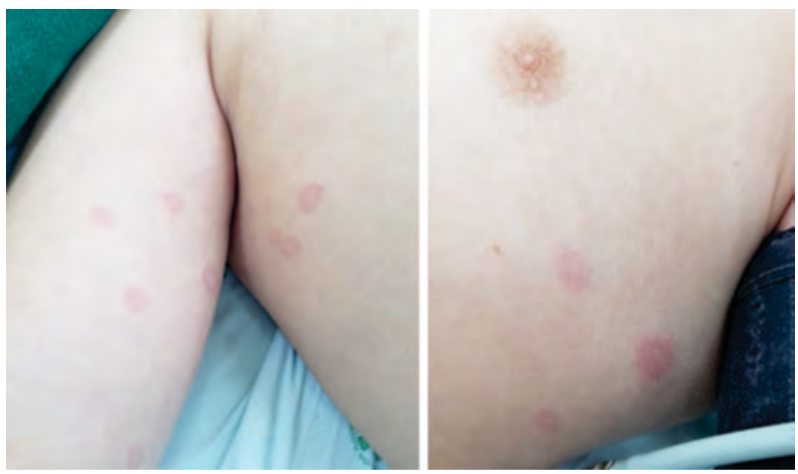

Fig 5 y 6 . Placas numulares eritematosas, bien delimitadas, en tronco y brazos.
Los datos de laboratorio con ligera leucocitosis y linfopenia de hasta 800, y marcadores inflamatorios elevados con PCR de hasta 237, Ferritina hasta un valor de 620, Transferrina de 115, Fibrinógeno de 800, Dímero D hasta 4080, VSR 58, Interleucina 6 elevada. Todos los cultivos tomados fueron negativos, así como otras determinaciones para Dengue, Epstein Barr, Citomegalovirus, Hepatitis A, B, y C, Herpes simple, Sífilis.

La radiografía de tórax presentaba áreas de condensación en ambas bases con predominio en segmentos posteriores y escaso derrame pleural bilateral.

La ecografía de abdomen con adenopatía de aspecto inflamatorio a nivel del mesenterio, en proyección periumbilical derecha, la mayor de $22 \times 16 \mathrm{~mm}$.

Tomografía de abdomen con numerosas adenopatías mesentéricas, sobre todo en fosa iliaca derecha de hasta $2 \mathrm{~cm}$ de diámetro. Fig. 7 y 8

La ecocardiografía mostro hiperrefringencia y tortuosidad de ambas arterias coronarias más marcado en el derecho.

Ante sospecha de Síndrome inflamatorio multisistémico (MIS-C) por antecedente de cuadro Covid19, historia clínica, examen físico y exámenes de laboratorio y estudios por imágenes compatibles recibe goteo de Inmunoglobulina a $2 \mathrm{gr} / \mathrm{kg}$ en su $1 \mathrm{er}$ día de internación, que se repite al 4to día por la persistencia de síntomas y signos con buena respuesta. Recibió Heparina de bajo peso molecular a dosis profiláctica por 9 días, además de Aspirina a $80 \mathrm{mg} / \mathrm{kg} /$ día, luego a $50 \mathrm{mg} / \mathrm{kp} /$ dosis completando 8 días.

Va de alta tras mejoría clínica, afebril, con indicación de AAS $250 \mathrm{mg}$ día, Amoxicilina + Trimetoprim/Sulfametoxaxol VO. Control a la semana con seguimiento multidisciplinario (Cardiología, Infectología y Reumatología). 

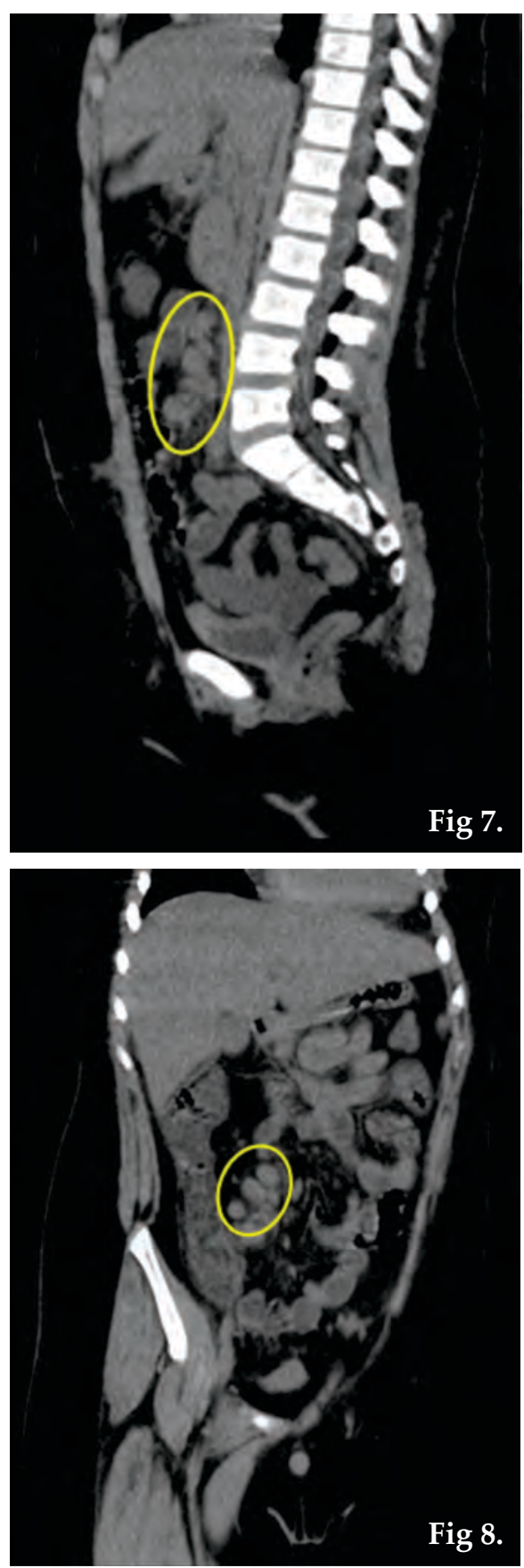

Fig 7 y 8. Adenopatías mesentéricas en fosa iliaca derecha. Marcadas con un círculo.

\section{DISCUSIÓN}

El presente caso es el primero reportado en el país.de síndrome inflamatorio multisistémico similar a la enfermedad de Kawasaki. De acuerdo al informe epidemiologico de la pandemia en el país, la afectación pediátrica es baja y muy leve.

El caso reportado es el primer paciente pediátrico que requirió ingreso a la UCI por presentar un shock toxico, con marcadores inflamatorios, reactantes de fase.

El paciente presentado fue remitido desde otro hospital donde le habían practicado una cirugía de abdomen por un cuadro abdominal agudo. El hallazgo operatorio informó la presencia de extensa inflamación de todos los órganos intrabdominales, incluido el apéndice que fue extirpado. La presentación abdominal de la infección del SARS $\mathrm{CoV} 2$ en niños fue reportada en una serie de casos en España, con acompañamiento mínimo o nulo de síntomas respiratorios $^{(8)}$.

El cuadro clínico y laboratorial es similar a los reportes de afectación mutisistémica con características de Enfermedad de Kawasaki (E.K.) reportado en Europa y EEUU ${ }^{(7,9)}$. La enfermedad e Kawasaki en sus presentaciones clásica o atípica puede presentarse con cuadros de Shock toxico. Esta enfermedad de etiología no bien definida, es más frecuente en niños menores de 5 años y se caracteriza por una gran inflamación que se inicia en el endotelio vascular, produciendo una vasculitis. Infecciones previas por números patógenos se han relacionado con la EK No se conoce con precisión si la infección y el estado de inflamación multisistémica producida por el or SARS Cov 2 pudiera disparar el desarrollo de la EK o el Síndrome multisistémico inflamatorio en niños (MIS C) observado en la pandemia es una entidad diferente a la EK por sus siglas en ingles. A diferencia de ésta, el MIS C afecta a niños mayores tal como el caso presentado ${ }^{(10,11)}$.

El paciente reportado había presentado la infección por SARS CoV-2 un mes antes de la aparición de los síntomas multisistémicos y al momento del inicio de este ya presentaba 3 pruebas negativas. Se realizó además pruebas serológicas mediante inmunohistoquímica a los 10 días del inicio de los síntomas la cual retorno IgG POSITIVO para SARS CoV2 e IgMnegativo. 


\section{REFERENCIAS}

1. Xu J, Zhao S, Teng T, Abdalla AE, Zhu W, Xie L, et al. Systematic Comparison of Two Animal-to-Human Transmitted Human Coronaviruses: SARS-CoV-2 and SARS-CoV. Viruses. 2020;12(2):244. doi: https://doi.org/ 10.3390/v12020244

2. Wang Y, Wang Y, Chen Y, Qin Q. Unique epidemiological and clinical features of the emerging 2019 novel coronavirus pneumonia (COVID-19) implicate special control measures. J Med Virol. 2020;92(6):568-576. doi: https://doi.org/10.1002/jmv.25748

3. Chan JF, Yuan S, Kok KH, To KK, Chu H, Yang J, et al. A familial cluster of pneumonia associated with the 2019 novel coronavirus indicating person-to-person transmission: a study of a family cluster. Lancet. 2020;395(10223):514-523. doi: https://doi.org/10.1016/S014 $0-6736(20) 30154-9$

4. Cao Q, Chen YC, Chen CL, Chiu CH. SARS-CoV-2 infection in children: Transmission dynamics and clinical characteristics. J Formos Med Assoc. 2020;119(3):670-673. doi: https://doi.org/10.1016/j.jfma.2020.02.009

5. Zhu H, Wang L, Fang C, Peng S, Zhang L, Chang G, et al. Clinical analysis of 10 neonates born to mothers with 2019nCoV pneumonia. Transl Pediatr. 2020;9(1):51-60. doi: https://doi.org/10.21037/tp.2020.02.06

6. Castagnoli R, Votto M, Licari A, Brambilla I, Bruno R, Perlini S, et al. Severe Acute Respiratory Syndrome Coronavirus 2 (SARS-CoV-2) Infection in Children and Adolescents: A Systematic Review. JAMA Pediatr. 2020;
Published online April 22. doi: https://doi.org/10.1001 /jamapediatrics.2020.1467

7. Verdoni L, Mazza A, Gervasoni A, Martelli L, Ruggeri M, Ciuffreda M, et al. An outbreak of severe Kawasaki-like disease at the Italian epicentre of the SARS-CoV-2 epidemic: an observational cohort study. Lancet. 2020;395(10239):1771-1778. doi: https://doi.org/10.1016/ S0140-6736(20)31103-X

8. Cabrero-Hernández M, García-Salido A, Leoz-Gordillo I, Alonso-Cadenas JA, Gochi-Valdovinos A, González Brabin A, et al. Severe SARS-CoV-2 Infection in Children With Suspected Acute Abdomen: A Case Series From a Tertiary Hospital in Spain. Pediatr Infect Dis J. 2020; Volume Online First. doi: https://doi.org/10.1097/INF.000 0000000002777

9. Jones VG, Mills M, Suarez D, Hogan CA, Yeh D, Segal JB, et al. COVID-19 and Kawasaki Disease: Novel Virus and Novel Case. Hosp Pediatr. 2020;10(6):537-540. doi: https://doi.org/10.1542/hpeds.2020-0123

10. Xu S, Chen M, Weng J. COVID-19 and Kawasaki disease in children. Pharmacol Res. 2020;159:104951. doi: https://doi.org/10.1016/j.phrs.2020.104951

11. Rowley AH. Understanding SARS-CoV-2-related multisystem inflammatory syndrome in children. Nat Rev Immunol. 2020; Published online May 25. doi: https://doi.org/10.1038/s41577-020-0367-5 